\title{
NOUVELLE
}

\section{Épimutations héréditaires}

\section{Le point chez l'homme}

Angélique Galvani, Déborah Bourc'his
Inserm U741, Université Denis Diderot Paris 7, 2, place Jussieu, 75251 Paris Cedex 05, France. bourchis@ijm.jussieu.fr

promoteur de $\mathrm{MLHI}$ est retrouvée dans $1 \%$ de ses spermatozoïdes : pour la première fois, la possibilité de transmission d'une épimutation était évoquée chez I'homme. Revirement de situation en 2007 : le même groupe analyse de nouveau l'état de méthylation des spermatozoïdes de ce patient, en incluant cette fois un contrôle crucial, SNRPN, un gène soumis à empreinte à méthylation maternelle et donc déméthylé dans les spermatozoïdes [8]. II apparaît que le faible pourcentage de méthylation observé précédemment était dû en fait à une contamination par des cellules somatiques, remettant en cause le possible maintien de cette épimutation lors de la spermatogenèse.

Une étude parallèle réalisée par les mêmes auteurs montre que l'épimutation pourrait en revanche être transmise maternellement; cette étude documente le cas d'une mère et son fils présentant la même hyperméthylation constitutionnelle de $\mathrm{MLHI}$ au niveau somatique [9]. Les spermatozoïdes du fils ne sont cependant plus porteurs de l'épimutation. Ces observations suggèrent un mode de transmission sexe-spécifique de l'épimutation $M L H I$, avec effacement lors du passage par la lignée germinale mâle, mais maintien lors du passage par la lignée germinale femelle.

\section{Maintien ou ré-écriture? Épimutation} ou mutation à effet épigénétique?

Pour des raisons éthiques évidentes, la présence de l'épimutation n'a pu être analysée dans les ovocytes de la patiente. Le mécanisme moléculaire à l'origine de cette transmission transgénérationnelle reste inconnu. Plusieurs hypothèses: (1) L’hyperméthylation a pu être réellement main- 
tenue lors de l'ovogenèse et est transmise telle quelle au fils; (2) I'hyperméthylation a été effacée puis rétablie lors de l'ovogenèse, à la manière d'un gène soumis à empreinte maternelle; ou bien (3) I'hyperméthylation a été effacée puis ré-imposée après fécondation. Dans tous les cas, on ne peut exclure l'existence d'un déterminisme génétique à l'origine de l'épimutation: un haplotype conférant une résistance à la déméthylation germinale ou favorisant la re-méthylation ovocytaire ou zygotique. Certes aucun polymorphisme de séquence n'a été associé à l'allèle hyperméthylé de MLHI. Cela n'exclut pas pour autant l'existence d'une mutation touchant des séquences régulatrices et qui induirait en cis la méthylation du promoteur, ou une mutation en trans touchant un gène codant pour une protéine intervenant dans la méthylation ou la déméthylation de I'ADN. Dans ce cas, d'autres séquences génomiques pourraient également présenter des anomalies de méthylation.

Le mode de transmission de l'épimutation $M L H I$ reste donc incertain. L'hyperméthylation est-elle réellement maintenue de façon autonome et sexe-spécifique, ou

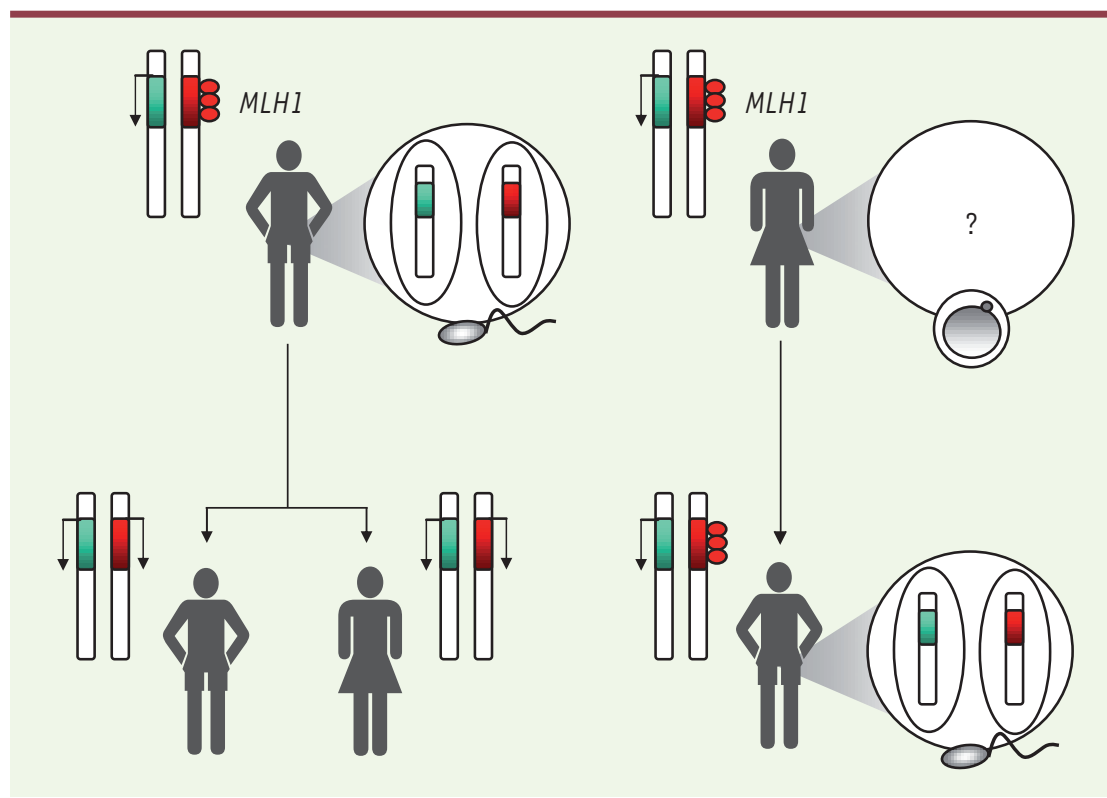

Figure 1. Transmission de l'épimutation MLH1. Le patient décrit dans [6], à gauche, présente une hyperméthylation (cercles rouges) au niveau du promoteur de $M L H I$ sur un des deux allèles, entraînant une perte d'expression du gène. Cette épimutation est perdue dans ses spermatozoïdes, traduisant un effacement de la méthylation lors de la spermatogenèse. La patiente décrite dans [9], à droite, a transmis l'épimutation à un de ses fils. Cependant, la présence de l'hyperméthylation au promoteur de MLHI n'a pas été déterminée dans ses ovocytes, laissant planer un doute sur le mode réel de transmission de l'épimutation. Chez le fils, l'épimutation est perdue dans les spermatozoïdes.

est-elle le reflet d'une mutation génétique affectant le statut de méthylation de MLHI ? Par analogie avec la souris, il serait tentant de spéculer que l'insertion d'un rétrotransposon à proximité du locus MLHI pourrait être responsable de la résistance variable à la reprogrammation germinale. Seul le séquençage complet du génome de ces patients permettrait de valider l'existence d'épimutations héréditaires dans l'espèce humaine. II en reste néanmoins que des altérations épigénétiques peuvent prédisposer au cancer et être transmises, avec une étiologie génétique ou non, au cours des générations. $\diamond$

Heritable epimutations:

a case study in humans

\section{RÉFÉRENCES}

1. Dolinoy DC, Das R, Weidman JR, Jirtle RL. Metastable epialleles, imprinting, and the fetal origins of adult diseases. Pediatr Res 2007; 61 : R30-7.

2. Prouteau $M$, Colot V. Contrôles épigénétiques, développement et variation génétique naturelle chez les plantes. Med Sci (Paris) $2005 ; 23: 422-7$.

3. Trasler JM. Gamete imprinting : setting epigenetic patterns for the next generation. Reprod Fertil Dev $2006 ; 18: 63-9$.

4. Morgan HD, Sutherland HG, Martin DI, Whitelaw $\varepsilon$ Epigenetic inheritance at the agouti locus in the mouse. Nat Genet $1999 ; 23: 314-8$.

5. Hajkova P, Erhardt $S$, Lane $N$, et al. Epigenetic reprogramming in mouse primordial germ cells. Mech Dev 2002; $117: 15-23$.

6. Suter MC, Martin DIK, Ward RL. Germline epimutations of MLH1 in individuals with multiple cancers. Nat Genet $2004 ; 36: 497-501$.

7. Duval A, Hamelin R. Réparation des erreurs de réplication, microsatellites et cancer. Med Sci (Paris) $2003 ; 19: 55-62$.

8. Hitchins MP, Ward RL. Erasure of MLHl methylation in spermatozoa- implications for epigenetic inheritance. Nat Genet $2007 ; 39$ : 1289.

9. Hitchins MP, Wong JJL, Suthers G, et al. Inheritance of a cancer-associated $\mathrm{MLHl}$ germ-line epimutation. N Eng J Med 2007 ; 356 : 697-705
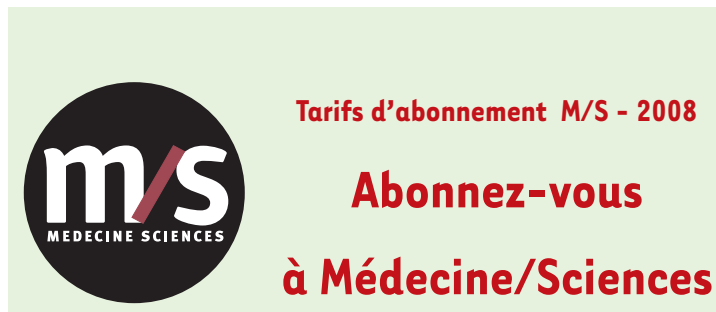
$>$ Grâce à $m / s$, vous vivez en direct les progrès des sciences biologiques et médicales

à Médecine/Sciences

\section{Bulletin d'abonnement} page 554 dans ce numéro de $\mathrm{m} / \mathrm{s}$
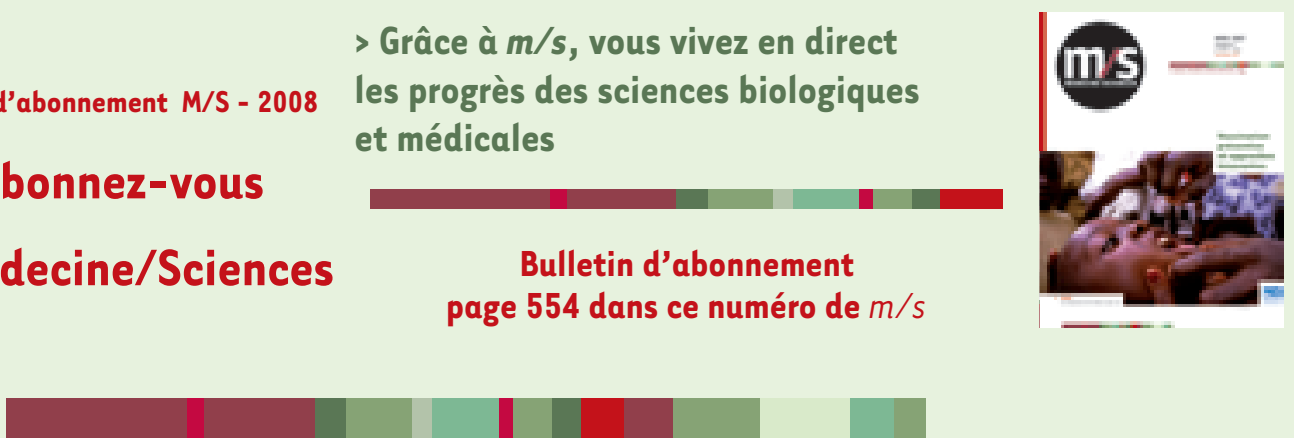